\title{
Erratum: A Parabolic Quasilinear Problem for Linear Growth Functionals
}

(Rev. Mat. Iberoamericana 18 (2002), no. 1, 135-185)

\section{Fuensanta Andreu, Vicent Caselles and José Manuel Mazón}

\begin{abstract}
We give the correct proof of Lemma 3.6 of the paper $A$ Parabolic Quasilinear Problem for Linear Growth Functionals (Rev. Mat. Iberoamericana 18 (2002), no. 1, 135-185).
\end{abstract}

Following [2], let

$$
X(\Omega)=\left\{z \in L^{\infty}\left(\Omega, \mathbb{R}^{N}\right): \operatorname{div}(z) \in L^{1}(\Omega)\right\} .
$$

In [2], the weak trace on $\partial \Omega$ of the normal component of $z \in X(\Omega)$ is defined. More precisely, it is proved that there exists a linear operator $\gamma: X(\Omega) \rightarrow L^{\infty}(\partial \Omega)$ such that

$$
\begin{gathered}
\|\gamma(z)\|_{\infty} \leq\|z\|_{\infty} \\
\gamma(z)(x)=z(x) \cdot \nu(x) \quad \text { for all } x \in \partial \Omega \text { if } z \in C^{1}\left(\bar{\Omega}, \mathbb{R}^{N}\right) .
\end{gathered}
$$

We shall denote $\gamma(z)(x)$ by $[z, \nu](x)$.

Assuming that $\partial \Omega$ is of class $C^{1}$, it is proved in [3] that if $x_{0} \in \partial \Omega$ is a Lebesgue point of the function $[z, \nu]$, then

$$
[z, \nu]\left(x_{0}\right)=\lim _{\rho \rightarrow 0} \lim _{r \rightarrow 0} \frac{1}{2 r w_{N-1} \rho^{N-1}} \int_{C_{r, \rho}\left(x_{0}, \nu\left(x_{0}\right)\right)} z(y) \cdot \nu\left(x_{0}\right) d y,
$$

$C_{r, \rho}(x, \alpha)$ being the cylinder defined by

$$
C_{r, \rho}(x, \alpha)=\left\{y \in \mathbb{R}^{N}:|(y-x) \cdot \alpha|<r,|(y-x)-[(y-x) \cdot \alpha] \cdot \alpha|<\rho\right\} .
$$

2000 Mathematics Subject Classification: 35K65, 35K55, 47H06, 47H20.

Keywords: Linear growth functionals, nonlinear parabolic equations, accretive operators, nonlinear semigroups. 
Our purpose is to give the correct proof of the following Lemma.

Lemma 1.1 ([1, Lemma 3.6])

i) Let $u_{n} \in B V(\Omega) \cap L^{2}(\Omega)$ and $z \in X(\Omega)$. Suppose that

$$
\mathbf{a}\left(x, \nabla u_{n}\right) \rightarrow z \quad \text { weakly }{ }^{*} \text { in } L^{\infty}\left(\Omega, \mathbb{R}^{N}\right)
$$

and

$$
\operatorname{div}\left(\mathbf{a}\left(x, \nabla u_{n}\right)\right) \rightarrow \operatorname{div} z \quad \text { weakly in } L^{2}(\Omega) .
$$

Then

$$
|z(x) \cdot \nu(x)| \leq f^{0}(x, \nu(x)) \quad \text { a.e. in } \partial \Omega \text {. }
$$

ii) Let $u_{n} \in W^{1,2}(\Omega)$. Let $\mathbf{a}_{n}(x, \xi)=\mathbf{a}(x, \xi)+\frac{1}{n} \xi$. Suppose that

$$
\mathbf{a}_{n}\left(x, \nabla u_{n}\right) \rightarrow z \quad \text { weakly in } L^{2}\left(\Omega, \mathbb{R}^{N}\right)
$$

and

$$
\operatorname{div}\left(\mathbf{a}_{n}\left(x, \nabla u_{n}\right)\right) \rightarrow \operatorname{div} z \quad \text { weakly in } L^{2}(\Omega)
$$

Then

$$
\begin{gathered}
{\left[\mathbf{a}_{n}\left(x, \nabla u_{n}\right), \nu(x)\right] \rightarrow[z, \nu(x)] \quad \text { weakly in } W^{1 / 2,2}(\partial \Omega)^{*} \text { and }} \\
|[z(x), \nu(x)]| \leq f^{0}(x, \nu(x)) \quad \text { a.e. in } \partial \Omega .
\end{gathered}
$$

To prove it, let us recall the following result, which corresponds to Lemma 3.7 in [1]. We notice that its proof is independent of Lemma 3.6. 
Lemma 1.2 ([1, Lemma 3.7]) Suppose that any of the assumptions of Lemma 3.6 hold. Moreover we assume that

$$
u_{n} \rightarrow u \text { in } L^{2}(\Omega) \text { and }\left\|u_{n}\right\|_{B V} \text { is bounded, }
$$

Then

$$
z(x)=\mathbf{a}(x, \nabla u(x)) \quad \text { a.e. } x \in \Omega .
$$

Proof of Lemma 1.1. Since both proofs are based on similar arguments, we shall only prove $i i)$. For the proof of (1.11) we refer to [1]. Let us prove (1.12). By (1.2), we have

$[z, \nu](x)=\lim _{\rho \rightarrow 0} \lim _{r \rightarrow 0} \frac{1}{2 r w_{N-1} \rho^{N-1}} \int_{C_{r, \rho}(x, \nu(x))} z(y) \cdot \nu(x) d y \quad \mathcal{H}^{N-1}$-a.e. on $\partial \Omega$.

On the other hand, by assumption $\left(H_{5}\right)$,

$$
\mathbf{a}(x, \xi) \cdot \eta \leq f^{0}(x, \eta)
$$

for all $\xi, \eta \in \mathbb{R}^{N}$, and all $x \in \bar{\Omega}$. Then, by 1.2 , we have

$$
z(y) \cdot \nu(x)=\mathbf{a}(y, \nabla u(y)) \cdot \nu(x) \leq f^{0}(y, \nu(x)) .
$$

Finally, since $f^{0}(\cdot, \xi)$ is continuous in $\bar{\Omega}$ for all $\xi \in \mathbb{R}^{N}$, using (1.15), (1.16), we get

$$
\begin{aligned}
&|[z, \nu](x)| \leq \lim _{\rho \rightarrow 0} \lim _{r \rightarrow 0} \frac{1}{2 r w_{N-1} \rho^{N-1}} \int_{C_{r, \rho}(x, \nu(x))}|z(y) \cdot \nu(x)| d y \\
& \leq \lim _{\rho \rightarrow 0} \lim _{r \rightarrow 0} \frac{1}{2 r w_{N-1} \rho^{N-1}} \int_{C_{r, \rho}(x, \nu(x))} f^{0}(y, \nu(x)) d y=f^{0}(x, \nu(x)), \\
& \mathcal{H}^{N-1}-\text { a.e. on } \partial \Omega .
\end{aligned}
$$

\section{References}

[1] Andreu, F., Caselles, V. and Mazón, J. M.: A Parabolic Quasilinear Problem for Linear Growth Functionals. Rev. Mat. Iberoamericana 18 (2002), no. 1, 135-185.

[2] Anzellotti, G.: Pairings between measures and bounded functions and compensated compactness. Ann. Mat. Pura Appl. (4) 135 (1983), 293-318. 
[3] Anzellotti, G.: Traces of bounded vector fields and the Divergence Theorem. Unpublished paper.

Recibido: 22 de enero de 2008

Fuensanta Andreu

Dept. de Matemática Aplicada Universitat de Valencia Dr. Moliner 50, 46100 Burjassot, Spain

Fuensanta.Andreu@uv.es

Vicent Caselles

Universitat Pompeu-Fabra

Dept. de Tecnologia

La Rambla 30-32, 08002 Barcelona, Spain

Vicent.Caselles@tecn.upf .es

José M. Mazón

Dept. de Análisis Matemático Universitat de Valencia Dr. Moliner 50, 46100 Burjassot, Spain

Mazon@uv.es 